\title{
La valoración de los maestros sobre la utilización didáctica de las ideas de los alumnos
}

\section{The opinion of the teachers on the educational use of students' ideas}

\author{
Rosa MARTÍN DEL POZO y Ángel DE-JUANAS OLIVA \\ Universidad Complutense de Madrid y Universidad Nacional de Educación a Distancia
}

Recibido: Octubre 2012

Aceptado: Diciembre 2012

\section{Resumen}

Uno de los indicadores de calidad en la enseñanza de las ciencias es la utilización didáctica que se hace de las ideas de los alumnos. Tanto en el currículo de Primaria como en el del Grado de Maestro se hace referencia a ello. Ahora bien, los maestros en activo y en formación ¿le dan tanta importancia? ¿utilizan mucho esa capacidad en su práctica habitual? ¿se consideran adecuadamente formados para ejercitarla? ¿qué significan para un maestro las ideas de los alumnos? Se abordan estas cuestiones a partir de los datos de cuestionarios y entrevistas con diferentes muestras de maestros, en formación y en activo. Los resultados indican que los maestros no otorgan a una especial importancia a esta capacidad, utilizan las ideas de los alumnos solo al iniciar el tema, no se consideran muy formados para ello y declaran una gran diversidad de significados: las ideas son opiniones, experiencias, errores, nivel de conocimientos o incluso una forma de motivación y participación. Todo ello está muy alejado de los planteamientos con los que la Didáctica de las Ciencias considera las ideas de los alumnos. Finalmente, se discuten las implicaciones que resultan para la formación inicial de maestros.

Palabras clave: formación de profesores, ejercicio profesional, cognición, enseñanza primaria.

\section{Abstract}

One of the indicators of quality in science education is the teaching that makes use of the students' ideas. Both the primary curriculum as in the Master's Degree refers to it. However, existing teachers and training do you give so much importance? Do you use a lot of that ability

\footnotetext{
${ }^{1}$ Esta publicación es resultado parcial del proyecto "Competencias profesionales y Formación del Profesorado. Estudio con Maestros en Formación y en Activo de la CM" (CCGO6UCM/HUM-1034) financiado por la Comunidad de Madrid y la Universidad Complutense de Madrid y desarrollado por el Grupo de Investigación Interdisciplinar sobre Competencias Profesionales Docentes de la UCM, del que los autores forman parte.
} 
in practice? Are they considered adequately trained to exercise it? What do they mean for a teacher the students' ideas? These questions are addressed using data from questionnaires and interviews with different samples of teachers in training and active. The results indicate that teachers do not give special importance to this capability, using the ideas of students just starting the topic, not considered highly trained to use it and report a wide range of meanings: the ideas are opinions, experiences, errors, level of knowledge or even a form of motivation and participation. All this is far removed from the approach to the Science Education considers students' ideas. Finally, we discuss the implications that are given for the initial training of teachers.

Keywords: teacher education, career development, cognition, primary education.

La mejora de la formación inicial del profesorado, en tiempos de reformas educativas, es considerada como una pieza clave para que la educación responda a las demandas sociales. Una de las dificultades para lograr esa mejora es la falta de vínculos entre la formación y las necesidades que plantea el ejercicio profesional (Zabalza, 2004). Por ello, consideramos necesario indagar sobre la visión que los maestros en formación y en activo tienen sobre determinados elementos que resultan clave para la enseñanza y discutir sus implicaciones en la formación inicial. Uno de estos elementos, en el área de Conocimiento del Medio, es la utilización de las ideas de los alumnos en la enseñanza y aprendizaje de las ciencias.

Varios autores (Joram y Gabriele, 1998; Jones, Carter y Rua, 1999; Meyer et al., 1999; Morrison y Lederman, 2003) señalan que la utilización didáctica las ideas de los alumnos constituye una herramienta importante para transformar el conocimiento del profesor, ya que aumenta su conocimiento del contenido y también de su enseñanza, es decir, su conocimiento didáctico del contenido (Shulman, 1987). Por otra parte, la predisposición didáctica de considerar las ideas de los alumnos, ayuda a mantener a los profesores apartados del modelo transmisivo de enseñanza de las ciencias. Tanto es así que estudios sobre las concepciones y prácticas de futuros maestros en el contexto de programas de formación dirigidos hacia el cambio conceptual, revelan que los cambios identificados están relacionados con un rediseño del papel desempeñado por las ideas de los alumnos en sus concepciones sobre el aprendizaje y la enseñanza (Joram y Gabriele, 1998; Meyer et al., 1999; Morrison y Lederman, 2003; Porlán et al., 2011)

Esta importancia se corrobora en el estudio realizado por la Agencia Nacional de Evaluación de la Calidad y Acreditación (2004) con 180 formadores de 18 universidades españolas. En este trabajo se propusieron 23 competencias específicas para ser maestro, siendo la más valorada por los participantes el "Conocimiento de los contenidos que hay que enseñar, comprendiendo su singularidad epistemológica y la especificidad de su didáctica" (p.92). En concreto, en ciencias (dentro del área de Conocimiento del Medio) se valoró específicamente:

"Saber reconocer la diversidad de los alumnos y explicitar su conocimiento, situar éste en relación al conocimiento científico y diseñar o escoger intervenciones didácticas para facilitar el desarrollo del conocimiento científico (3.3 sobre 4). 
"Conocer las características de las principales dificultades en el aprendizaje-enseñanza de las ciencias experimentales, así como las particularidades más usuales del conocimiento de los alumnos (conocimiento previo) sobre los diversos temas del área de las ciencias experimentales y su influencia en el aprendizaje" (3.2 sobre 4).

Por su parte, en los requisitos para la verificación de los títulos universitarios oficiales que habilitan para el ejercicio de la profesión de Maestro en Educación Primaria (Orden ECI/3857/2007) se señala que los estudiantes de Magisterio deben adquirir la siguiente competencia: "Conocer las áreas curriculares de la Educación Primaria, la relación interdisciplinar entre ellas, los criterios de evaluación y el cuerpo de conocimientos didácticos en torno a los procedimientos de enseñanza y aprendizaje respectivos." Más concretamente, en el Módulo Didáctico y Disciplinar (Ciencias Experimentales, Sociales, Matemáticas, Lenguas, Educación Musical, Plástica y Visual, y Educación Física) se dice, por ejemplo, que debe adquirirse la siguiente competencia específica:

"Comprender los principios básicos y las leyes fundamentales de las ciencias experimentales (Física, Química, Biología y Geología). Conocer el currículo escolar de estas ciencias. Plantear y resolver problemas asociados con las ciencias a la vida cotidiana. Valorar las ciencias como un hecho cultural. Reconocer la mutua influencia entre ciencia, sociedad y desarrollo tecnológico, así como las conductas ciudadanas pertinentes, para procurar un futuro sostenible. Desarrollar y evaluar contenidos del currículo mediante recursos didácticos apropiados y promover la adquisición de competencias básicas en los estudiantes".

También, en el currículo de Primaria (Orden ECI/2211/2007), en las orientaciones metodológicas para el área de Conocimiento del Medio se afirma:

"Partir en cualquier actividad de las ideas de los alumnos, de sus conocimientos y experiencias. En esta área tan relacionada con la experiencia, niños y niñas han adquirido en el contacto diario con el medio que le rodea muchos conocimientos de los que es necesario partir porque forman su bagaje personal. Estos preconceptos o ideas previas son numerosos en el área, en su mayoría se adquieren paralelamente a la adquisición del lenguaje en el propio entorno familiar, por lo que es necesario que el docente los conozca bien para ajustar la acción didáctica".

"En esta etapa es especialmente importante planificar actividades concretas antes y durante el proceso de enseñanza y aprendizaje de cualquier contenido para asegurarnos que esas ideas se van modificando".

Por tanto, las ideas de los alumnos son un elemento esencial para el aprendizaje y la enseñanza de las ciencias. La pregunta es si todo ello es compartido y utilizado por los maestros.

Dentro de esta problemática, nuestra aportación se centra en la descripción y análisis de la valoración que los maestros en formación y en activo hacen de las ideas de los alumnos. Para ello, primeramente haremos referencia a diferentes planteamientos sobre las ideas de los alumnos que han servido de fundamento para este estudio. 


\section{Fundamentación}

Las concepciones de los alumnos constituyen el objeto de estudio de una de las líneas de investigación más desarrolladas en Didáctica de las Ciencias (y en otras didácticas específicas) por su volumen, variedad e implicaciones para la enseñanza y la formación del profesorado. En estos trabajos se ha ofrecido información no solo sobre el contenido de tales ideas sino también sobre cuál puede ser su origen, cómo están organizadas y con qué estrategias didácticas pueden cambiar o evolucionar (Posada, 2000; Furió et al., 2006).

Como señala Cubero (2005), los investigadores en Didáctica de las Ciencias se han referido al hecho de que los alumnos desarrollan explicaciones propias sobre el mundo físico-natural con una gran cantidad de términos. En este sentido, la polisemia del lenguaje para designar este "saber" no deja de ser un reflejo de diferentes enfoques sobre la naturaleza, cambio y utilización en el proceso de enseñanza-aprendizaje de los conocimientos de los alumnos. No obstante, podemos decir que estos estudios se enmarcan en un planteamiento general del aprendizaje de orientación constructivista, en el que aprender ciencias consiste en elaborar modelos que permiten interpretar el mundo, en constante interacción entre lo que ya sabemos y la nueva información. Diferentes autores (Driver et al., 1989; 1999; Pozo et al., 1991; Oliva, 1999a; 1999b; Cubero, 2005) coinciden en la existencia de una serie de características generales de las concepciones de los alumnos, que podemos resumir:

- Son construcciones personales de los alumnos elaboradas de un modo más o menos espontáneo en su interacción con el mundo social y natural, pero compartidas por diferentes grupos; es decir, poseen un cierto carácter transcultural.

- El hecho de que existan patrones comunes no significa ni mucho menos que todos los alumnos de una clase tengan las mismas ideas sobre algo. Una de las características de las ideas de los alumnos que más implicaciones didácticas tiene es su diversidad en un mismo grupo de alumnos, lo cual obliga a plantearnos la necesidad de encontrar estrategias didácticas que permitan a los alumnos que sus ideas "mejoren" desde el nivel en el que se encuentra cada uno.

- Suelen ser incoherentes desde el punto de vista del conocimiento científíco y del conocimiento escolar, pero no desde el punto de vista del alumno puesto que le son útiles para explicar y predecir los fenómenos cotidianos. Así, por ejemplo, las ideas que utilizan los alumnos en una determinada situación pueden variar dependiendo del contexto.

- Son bastante estables y persistentes, a pesar de la enseñanza recibida. Esto es más evidente en la medida en que las ideas transmitidas están más alejadas de la intuición.

- Por lo que respecta al cambio de las ideas, parece que va más allá de la mera sustitución de unas ideas puntuales por otras científicamente más aceptables; más bien, se entiende como un proceso gradual de enriquecimiento y reestructuración 
de las estructuras conceptuales de los alumnos, de su manera de ver el mundo, y existe un cierto paralelismo con la historia de la ciencia.

- Se manifiestan a través del lenguaje oral, escrito y gráfico, pero esencialmente están implícitas en las actividades que los alumnos realizan.

- No parecen ser ideas aisladas sino que constituyen estructuras, esquemas, marcos, teorías personales o sistemas de ideas, pero con una escasa relación jerárquica.

En la construcción de estructuras conceptuales más complejas a partir de otras más simples, parece haber tres procesos fundamentales. Estos procesos son el fundamento de las estrategias didácticas para hacer evolucionar las ideas de los alumnos, y son los siguientes (Pozo y Gómez Crespo, 1998):

- La explicitación progresiva de las ideas, debido al carácter implícito de buena parte de ellas, tratando de formalizarlas al máximo para favorecer los procesos de reestructuración.

- La reestructuración, como proceso más profundo de cambio conceptual (actitudinal y metodológico), admitiéndose que pueden darse otros cambios más débiles, de enriquecimiento y ajuste de las ideas ya existentes.

- La integración jerárquica de las ideas de los alumnos en las ideas que consideremos deseables, teniendo en cuenta que éstas tendrán mayor capacidad de generalización, una estructura conceptual más compleja y un mayor poder explicativo.

Así, según Giordan y Vecchi (1995), las ideas de los alumnos corresponden a una activación de lo que construyeron como resultado de sus experiencias para explicar, predecir, o actuar ante una demanda específica. Durante esta activación, los alumnos construyen una red de análisis de la realidad que les permite comprender el mundo circundante, hacer frente a nuevos problemas, e interpretar las nuevas situaciones. Del mismo modo, Von Glaserfeld (1993) afirma que el conocimiento es el resultado de una acción constructiva y no puede ser simplemente "transferido" a un receptor. Por lo tanto, el desarrollo de nuevos conocimientos y procedimientos implica una interacción activa entre la construcción previa de la persona y la nueva información que emerge de las situaciones vividas. Entonces, las ideas de los alumnos son cruciales para cualquier proceso de aprendizaje.

No obstante, según Giordan (1989), hay diferentes planteamientos sobre las ideas de los alumnos, muy relacionados con los modelos didácticos. Así, podemos diferenciar tres visiones respecto a las ideas de los alumnos. Una, relacionada con un modelo didáctico de corte tradicional, en la que las ideas de los alumnos no tienen valor epistemológico, no se considera que tengan ideas propias, espontáneas, sino se conciben como el recuerdo, el "poso" que les queda de los contenidos ya explicados en el aula. Esta visión suele constituir el nivel inicial de los planteamientos de muchos maestros. Otra visión, de nivel intermedio, también muy frecuente, admite que los alumnos tienen ideas propias pero éstas son erróneas. El profesor necesita saberlas para subsanarlas y sustituirlas por el conocimiento verdadero (científico). En el nivel que actúa como referente en los procesos formativos, las ideas de los alumnos constituyen 
un conocimiento alternativo al científico-escolar, con cierta coherencia interna, no son necesariamente explícitas, su devenir puede ser diferente para distintos dominios y su evolución ocurre a través de un proceso mediante el cual las representaciones implícitas se reelaboran en formatos más explícitos y más estables. Este nivel de referencia es coherente con el constructivismo didáctico, que lo concibe como un conocimiento alternativo al escolar (García, Mateos y Vilanova, 2011).

En definitiva, de acuerdo con Treagust y Duit (2008), la investigación didáctica durante las últimas tres décadas ha demostrado que los alumnos van a clase de ciencias con ideas acerca de los fenómenos físico-naturales, que no están en consonancia con puntos de vista científicos. En otras palabras, hay consenso en admitir que no puede considerarse a los alumnos como una tabla rasa a la que llenar de contenidos. Por lo tanto, los profesores tienen que darse cuenta de que sus alumnos ya tienen conocimientos propios sobre los contenidos a enseñar, que son alternativos al conocimiento que se quiere enseñar y suponen verdaderos obstáculos para la comprensión conceptual.

Sin embargo, hay pocas investigaciones que informen de cómo los profesores piensan y actúan en relación a las ideas de los alumnos. Por ejemplo, Morrison y Lederman (2003), hallaron que ninguno de los participantes en su estudio mencionó el uso de estrategias para detectar las ideas de los alumnos en el aula, a pesar de que consideraron importante diagnosticarlas. Al analizar el conocimiento profesional del profesor, estos autores identificaron que un solo sujeto, con una mayor experiencia en la enseñanza, tenía un amplio conocimiento acerca de las concepciones previas que los alumnos traen al aula. La opinión predominante es que las ideas de los alumnos se toman como algo a eliminar, en la cual los pensamientos incorrectos deben ser sustituidos por el conocimiento correcto (López, 1994; Giordan y Vecchi, 1995; Park et al 2010; Porlán et al., 2011). No obstante, en el estudio de Haefner y Zembal-Saul (2004), encontraron que a pesar de que, al final del proceso de formación, los futuros profesores investigados no han desarrollado un profundo entendimiento del papel de las ideas de los alumnos en el aprendizaje, ellos reconocen que los alumnos tienen una gran variedad de ideas sobre los contenidos de ciencias, y estas ideas podrían ser útiles en la enseñanza.

\section{Diseño y metodología}

El problema de la investigación puede formularse como sigue: ¿Qué valoración hacen los maestros, en formación y en activo, de las ideas de los alumnos? Para abordarlo, el estudio se realiza en dos momentos. Primero se propone a la muestra de maestros en activo y en formación, mediante un cuestionario, un conjunto de indicadores de competencias fundamentales y se trata de averiguar cómo las valoran desde el punto de vista de su importancia para la profesión, de la utilización en la práctica y de la formación inicial recibida. En este trabajo nos referiremos prioritariamente a las ideas de los alumnos. En segundo lugar, se trata de que algunos de los maestros en activo encuestados, a través de una entrevista, expongan su visión de las ideas de los alumnos considerando esos mismos aspectos. 


\section{Objetivos}

- Describir y analizar la valoración sobre las ideas de los alumnos de una muestra de futuros maestros de Primaria, mediante un cuestionario.

- Describir y analizar la valoración sobre las ideas de los alumnos de una muestra amplia de maestros de Primaria, mediante un cuestionario.

- Comparar ambas muestras.

- Describir y analizar las creencias que una muestra reducida de maestros de Primaria declara, en una entrevista, sobre las ideas de los alumnos.

\section{Participantes}

La muestra general seleccionada está constituida por 343 maestros en activo que contestaron al cuestionario enviado a los 85 centros de Educación Primaria de la Comunidad de Madrid (España), en su mayoría de carácter público (91,7\%), que tenían un acuerdo con la Facultad de Educación de la UCM para la realización de sus prácticas docentes. La edad media de los sujetos supera los 45 años. Prácticamente tres cuartas partes de la muestra son mujeres $(72,4 \%)$. Casi la totalidad son funcionarios de carrera con más de 20 años de servicio. En su mayoría ejercen como maestros generalistas $(65,4 \%)$ y al mismo tiempo, más de la mitad, como tutores de su grupo de clase $(54,7 \%)$.

De esta muestra se entrevistaron a 48 maestros en activo que expresaron su deseo de colaborar más activamente. Esta sub-muestra tiene características semejantes a las de la muestra general.

El grupo de futuros maestros elegido estaba realizando el Prácticum en tercer curso de la especialidad de Educación Primaria. Sus opiniones se recogieron mediante un cuestionario de similares características que el que se aplicó a los maestros en activo por vía electrónica (www.ucm.es/info/grupocompetencias). Contestaron el cuestionario un total de 53 estudiantes. Todos eran mujeres, excepto un único varón, y la edad media del grupo se sitúa en torno a los 22 años.

\section{Instrumentos}

En primer lugar, se elaboró un cuestionario ad hoc para estudiar la valoración de los maestros en activo sobre un conjunto de 28 indicadores de competencias elaborados a partir de los diez dominios propuestos por Perrenoud (2004), con una escala tipo Likert, cuyas puntuaciones estaban comprendidas entre 0 y 3 (nada, poco, bastante y mucho) y en relación a los cuales había que dar una valoración de: importancia para la profesión docente; utilización en la práctica (en condicional para los maestros en formación); y valoración de la formación inicial recibida en la universidad.

Para los análisis estadísticos de las respuestas a los 28 ítems del cuestionario se utilizó el paquete estadístico SPSS 15.0. El coeficiente de fiabilidad de esta parte del cuestionario, se calculó mediante el alpha de Cronbach, y fue de 0.9308 en el caso de 
los maestros en activo y 0.927 en el de los maestros en formación. El indicador que analizamos en este caso, se formuló como sigue: Tengo en cuenta las ideas de mis alumnos, aunque sean erróneas, y las utilizo para su aprendizaje.

En segundo lugar, se realizaron 48 entrevistas individuales a otros tantos maestros en activo. La entrevista se planteó, fundamentalmente, sobre la valoración de los resultados más importantes del cuestionario. En el presente estudio nos referiremos exclusivamente a su valoración sobre las ideas de los alumnos.

Para analizar el contenido de las entrevistas se siguió un procedimiento basado en el análisis de contenido de Bardin (1977):

- Transcripción de las grabaciones de las 48 entrevistas realizadas.

- Codificación de los datos obtenidos en las entrevistas mediante un código numérico. Este código refleja la letra inicial del entrevistador, seguido del número de la entrevista, así como del número de cuestionario que el entrevistado respondió en el estudio previo a la entrevista. Por ejemplo: R.2 -128. Es la segunda entrevista realizada por el entrevistador $\mathrm{R}$ al maestro que cumplimentó el cuestionario 128.

- Establecimiento de Unidades de Información según el protocolo de la entrevista. Dichas unidades son las respuestas de los entrevistados a la formación recibida en cada una de las competencias consideradas, así como a la cuestión relativa a la valoración de la formación inicial.

- Reformulación de las Unidades de Información en Proposiciones de Síntesis, como paso previo a la interpretación, es decir, con un muy bajo nivel de inferencia.

- Finalmente, para la interpretación de las Proposiciones de Síntesis, se acordaron en el grupo de investigación, las diferentes categorías en las respuestas de los entrevistados que, por tanto, se establecieron a posteriori.

\section{Resultados}

\section{Valoración de las competencias propuestas en el cuestionario}

La utilización de las ideas de los alumnos ocupa de las últimas posiciones en la valoración de su importancia para la profesión (puesto 21) y utilización en la práctica docente (puestos 19 y 20), de las 28 propuestas en el cuestionario, aunque los futuros maestros se consideran mejor formados (puesto 5) que los maestros en activo (puesto 13) (Gráfico 1). En este sentido, mientras que $59.2 \%$ de los maestros en formación se considera bastante o muy bien formado en este ámbito, sólo lo es para el $30.6 \%$ de los maestros en activo. 


\section{Maestros en activo}

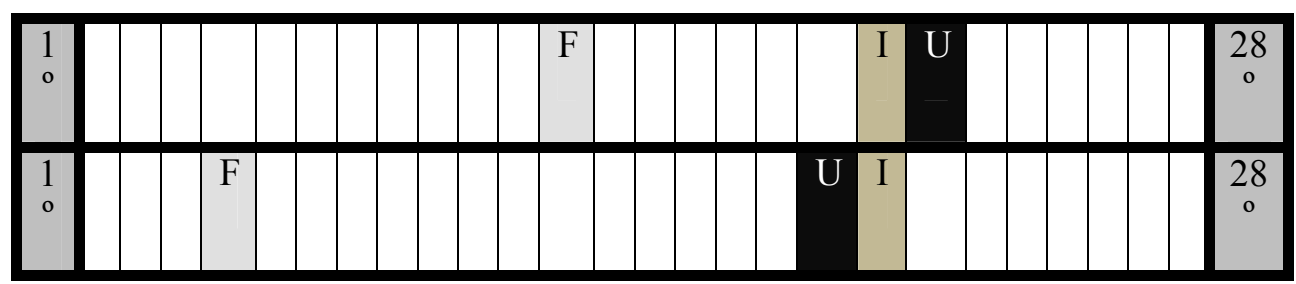

\section{Maestros en formación}

Gráfico 1. Puesto ocupado en la valoración de los 28 indicadores de competencias por la formación (F), utilización (U) e importancia (I) de las ideas de los alumnos.

La prueba de Kruskal-Wallis (prueba no paramétrica para $\mathrm{k}$ muestras independientes con un nivel de significación del $0.05 \%$ ) nos indica que entre los maestros en activo y en formación hay diferencias significativas en sus valoraciones (Tabla 1). No existen diferencias significativas entre las valoraciones de la importancia, utilización y formación en ninguna de las dos muestras (prueba de Wilcoxon, prueba no paramétrica para dos muestras relacionadas). Tampoco se detecta una correlación positiva entre la importancia dada a este indicador y los años de servicio de los maestros en activo. Así pues, a medida que van teniendo más experiencia no parece que le dan ni más ni menos importancia.

\begin{tabular}{|c|c|c|c|c|c|c|}
\hline \multirow{2}{*}{$\begin{array}{l}\text { Indicadores de } \\
\text { competencias }\end{array}$} & \multicolumn{2}{|c|}{ Importancia } & \multicolumn{2}{|c|}{ Utilización } & \multicolumn{2}{|c|}{ Formación } \\
\hline & $\begin{array}{l}\text { Maestros en } \\
\text { formación }\end{array}$ & $\begin{array}{c}\text { Maestros en } \\
\text { activo }\end{array}$ & $\begin{array}{l}\text { Maestros en } \\
\text { formación }\end{array}$ & $\begin{array}{c}\text { Maestros en } \\
\text { activo }\end{array}$ & $\begin{array}{l}\text { Maestros en } \\
\text { formación }\end{array}$ & $\begin{array}{c}\text { Maestros en } \\
\text { activo }\end{array}$ \\
\hline \multirow{3}{*}{$\begin{array}{l}\text { Tener en cuenta las } \\
\text { ideas de mis } \\
\text { alumnos y } \\
\text { utilizarlas para su } \\
\text { aprendizaje aunque } \\
\text { sean erróneas }\end{array}$} & 2.49 & 2.19 & 2.59 & 2.11 & 1.74 & 1.21 \\
\hline & $92.6 \%$ & $78.7 \%$ & $96.3 \%$ & $80.2 \%$ & $59.2 \%$ & $30.6 \%$ \\
\hline & \multicolumn{2}{|c|}{ Sig. .004 } & \multicolumn{2}{|c|}{ Sig. .000 } & \multicolumn{2}{|c|}{ Sig. .000 } \\
\hline
\end{tabular}

Tabla 1. Valores medios en importancia, utilización y formación recibida sobre las ideas de los alumnos, lugar de valoración, porcentajes de la suma de las valoraciones bastante (2) y mucho (3), y significación

Para poder tener una visión más amplia, diremos que, en el caso de los maestros en activo, la implicación de las familias en el aprendizaje de los hijos y la educación en valores ocupan los primeros puestos tanto en importancia como en utilización, mientras que los indicadores relacionados con las TIC, ocupan los últimos lugares (Pesquero et 
al., 2008). En el caso de los futuros maestros, es también la capacidad para implicar a las familias lo más valorado, pero lo menos es impulsar la participación de otras instituciones y agentes sociales en la vida del centro (González et al., 2010). En ambas muestras, la competencia para la que mejor han sido formados en la Universidad se refiere a los contenidos a enseñar, mientras que es en las TIC, para los maestros en activo, y la relación con las familias, para los futuros maestros, los ámbitos en los que se consideran menos formados (Pesquero et al., 2008; De-Juanas et al., 2009; González et al., 2010).

\section{Declaraciones sobre las ideas de los alumnos}

Importancia. E1 77\% de los entrevistados consideró fundamental tener en cuenta las ideas de los alumnos, aunque las razones no siempre tienen que ver con su importancia para el aprendizaje. Veamos algunas declaraciones al respecto:

Por supuesto, yo creo que sí y si realmente queremos hacer una educación liberal hace falta escucharlos y luego tu les reformulas: mira, creo que estás equivocado por esto y por esto. (E3-88)

A mi me parece que es importante partir de ellos, de sus inquietudes, de lo que piensan, de lo que quieren. Sí que me parece importante porque es una manera de motivarles y de que se involucren. (R5-141)

Conocer sus ideas implica saber cuál es el punto de partida para el aprendizaje. $Y$ aprender de las ideas, de los errores o con las ideas que ellos plantean, me parece muy importante. (M3-213)

Sin embargo, en el otro extremo hay un 19\% de maestros que no lo consideran fundamental, básicamente porque los alumnos no tienen ideas o son erróneas, o porque no tiene interés para transmitir los conocimientos. Ejemplos de ello son:

A estas edades, la verdad es que yo creo que de ellos parten pocas ideas, a estas edades (...) que son 7 añitos, el primer ciclo. Esto a lo mejor ya a partir de un $4^{\circ}, 5^{\circ}$ $o 6^{\circ}$ ya empiezan a despuntar ellos y a dar sus propias ideas. (R4-147)

Si, son importantes si están en lo cierto (se refiere a los alumnos), pero si no lo están, obviamente, no. (M1-212)

En la transmisión de conocimientos se puede ser un poco asertivo, pero los conocimientos están ahí, es el profesor el que los tiene que transmitir. (P4-308)

Además, parece que existen diferencias según los contenidos a los que se refieran. Por ejemplo:

Es importante sobre todo en determinadas materias. Por ejemplo, en Conocimiento del medio, y cuando habia Historia, pues en Historia. En otras materias, es más difícil. (Pi2-244) 
Más bien es en Conocimiento del Medio. En Matemáticas ellos aportan menos, y en Lengua, pues es más dificil también. (S8-95)

Significado y utilización. Los participantes ya advierten que una cosa es reconocer la importancia de las ideas de los alumnos y otra utilizarlas en el aula. Por ejemplo:

Todos reconocemos que las ideas de los alumnos son importantes, pero tendemos a ponerlo poco en práctica. (Pi1-246)

Yo sé que esa teoría es muy buena para el aprendizaje y es muy bueno para ellos. Pero algunas veces cuando sale la idea pienso ique tontería! y entonces me lo salto. (E1-155)

Además, se señalan las dificultades para tenerlas en cuenta:

Pues si, las tenemos en cuenta, pero a veces es que es imposible, porque son muy diversas (...) yo reconozco que no siempre tengo en cuenta las ideas de los alumnos por eso. Ya no es lo típico que teníamos antes de esa homogeneidad en el grupo, ahora no, ahora tienes veinte alumnos y a lo mejor tienes tres o cuatro grupos en el aula, para trabajar a distintos niveles. Entonces es dificil. (S4-98)

Los maestros declaran varias posiciones frente a las ideas de los alumnos:

a) Las ideas de los alumnos se consideran una expresión de sus intereses, opiniones y experiencias (31\%). Por ejemplo:

Les dejas hablar y a lo mejor basta con eso. Es la forma de que un niño se sienta importante en ese momento contando su experiencia o diciendo una palabra que se le ha ocurrido en ese momento. (E1-88)

Si a los niños les interesa, se trabaja sobre eso que les interesa, es decir, ¿para qué voy a trabajar algo que está marcado? si hay algo que está sucediendo en su historia, en su vida, en su presente, depende de las asignaturas, a lo mejor unas matemáticas, me dan menos pie a tener en cuenta sus ideas porque, pocas propuestas te pueden hacer. (S7-113)

b) Las ideas de los alumnos son errores que deben sustituirse por el conocimiento correcto $(23 \%)$. Por ejemplo:

Yo creo que fundamental para saber qué es lo que tienes que corregir y quitarles el error y decir: bueno pues esto no saben y necesito reforzar. (E2-140)

Siempre parto de lo que ellos conocen, y luego, ya sopeso, en función de cómo va el grupo, si esta erróneo, se modifica. (Pi8-199)

Conocerlas implica saber cuál es el punto de partida para el aprendizaje. Y que de aprender de las ideas, de los errores que ellos tienen, me parece muy importante. (M3-213) 
c) Las ideas de los alumnos como elemento de motivación y participación (19\%). Por ejemplo:

Yo desde luego les dejo que hablen mucho, que se expliquen, que me cuenten y que digan y entonces, cualquier sugerencia que hagan ellos, que tengan, cualquier idea me parece buena. Aunque son pequeños son bastante participativos y para los mayores me parece una idea muy buena. (S9-296)

Yo reconozco que no siempre tengo en cuenta las ideas de los alumnos, que deberíamos a lo mejor tenerlas más en cuenta y quizás así estarían más motivados y aprenderían mejor. (S4-98)

d) Las ideas de los alumnos se consideran como sus conocimientos previos, su nivel de partida, en definitiva, lo que recuerdan de cursos anteriores (15\%). Por ejemplo:

Ver lo que se da en $4^{\circ}$ o en $3^{\circ}$, a mi no me dice nada. Entonces se plantea, una serie de preguntas, para ver los conceptos que tienen ellos de partida. Ahora, por ejemplo, que estamos con el planeta Tierra, pues yo quiero saber si ya sabían lo que era una estrella, o si sabían lo que es el universo. (Pi1-246)

Y yo también creo que es esencial para tener en cuenta las ideas de los alumnos el tener un ambiente de trabajo, en la clase, agradable para todos y que te consideren cercano, y que tengan confianza en ti. (R2-128)

Por otra parte, los maestros que declaran utilizar las ideas de los alumnos lo hacen solo al iniciar el proceso de enseñanza-aprendizaje. Por ejemplo:

Al principio de cada tema, yo suelo hacerles un par de preguntitas, para ver que ideas tienen ellos del tema. Levantan la mano, participan, me contestan, y entonces, pues yo ya me hago una idea... a partir de ahí, pues entonces ya, empiezas a dar el contenido. (Pi1-246)

Solo en un caso, la utilización didáctica de las ideas de los alumnos parece ir más allá de la mera detección inicial:

Hay que buscar algún tipo de experimentación, donde puedan comprobar, desmontar su idea. Donde puedan deducir que su hipótesis no era buena. Pero sobre todo, hay que partir de su hipótesis. Lo que pasa que luego, vamos a comprobar esto. Entonces, le acercas la documentación que pueda ir desmontando esa hipótesis inicial. (Pi3-260)

Formación. Con anterioridad, cerca del $70 \%$ de los maestros encuestados se consideró poco o nada formado para utilizar las ideas de los alumnos. En las entrevistas, aunque la mayoría no hizo referencia a su formación (58\%), podemos decir que sigue manteniéndose la percepción de una formación inicial deficiente por el 17\% de los entrevistados, con argumentos que tienen que ver con la falta de formación metodológica, como puede apreciarse en estos casos: 
Yo creo que tampoco me formaron mucho. Sí, en la teoría, de decir: tenemos que partir de unos niños, que nos vamos a encontrar una clase y lo primero que tenemos que hacer es conocer a esos niños (...) Pero luego, el tener en cuenta las ideas de los alumnos, eso era, como (...) era mucho más cerrado, en mis tiempos, como que no, que no se trataba. (M5-264)

Pero a lo mejor muchas veces es porque no sabemos cómo. Por lo menos a mí me pasa, te incordia una idea, te rompe tus planes y entonces lo más fácil es decir: venga paso de ella. Es porque la formación no es la adecuada. (E1-155)

A lo mejor yo estoy muy equivocada y no sé llevar ese método (utilizar las ideas de los alumnos), quien lo sepa llevar, puede llevarlo, pero yo estoy más preparada para explicar los contenidos. (P3-92)

Sin embargo, para otros maestros (13\%) la formación inicial sí que cumplió su papel de poner de relieve la importancia para el aprendizaje. Por ejemplo:

Sí que es verdad que en la Facultad lo primero que te dicen es que hay que partir de las ideas de los alumnos, y de ahí ya se hace todo el tema, aunque yo en eso no estoy de acuerdo. (M4-340)

Yo es que he tenido la suerte de tener una muy buena profesora de Pedagogía, entonces sí que hacía mucho énfasis sobre todo en esto, en partir de los conocimientos de los chavales, siempre, nos hacía muchísimo hincapié. (P5-320)

También la propia experiencia es una modalidad formativa apuntada por algunos maestros $(8 \%)$ para adquirir esta capacidad profesional. Por ejemplo:

Sobre todo es la práctica en el aula. Los críos los pobres son como conejillos de indias muchas veces, sobre todo cuando ya llevas muchos años trabajando pues claro, ya sabes las cosas que te funcionan y aun así te pueden fallar. Pero ahora que soy joven pues ideas que me van dando un compañero $u$ otro... pues es ponerlo en práctica en el aula y ver qué es lo que funciona y lo que no. Cosas que dentro de esa idea te han funcionado se pueden mejorar y otras que las desechas directamente, dices: no me vale. (E2-140)

No obstante, también se admite la contribución de la formación inicial y la propia práctica:

Es que todo eso, yo te digo de verdad, que lo he aprendido cuando he empezado a trabajar. De todas formas, hay una asignatura que tuvimos en Magisterio, que era Psicología y, dependiendo también del profesor, o de la persona que te oriente un poco, yo aprendi también ahí cosas. (R7-204) 
En la Tabla 2 se resumen los diferentes tipos de respuestas obtenidas en las entrevistas.

\begin{tabular}{|l|c|c|}
\hline \multicolumn{1}{|c|}{ Importancia, utilización y formación } & $\mathrm{N}$ & $\%$ \\
\hline Es importante tener en cuenta las ideas de los alumnos & 37 & 77 \\
No es importante tener en cuenta las ideas de los alumnos & 9 & 19 \\
NC & 2 & 4 \\
\hline Las ideas de los alumnos como una expresión de sus intereses, opiniones y & 16 & 33 \\
experiencias & & \\
Las ideas de los alumnos como errores que deben sustituirse por el conocimiento & 11 & 23 \\
correcto & & \\
Las ideas de los alumnos como elemento de motivación y participación & 9 & 19 \\
Las ideas de los alumnos como sus conocimientos previos, su nivel de partida, en & & \\
definitiva, como lo que recuerdan de cursos anteriores & 7 & 15 \\
NC & 5 & 10 \\
\hline No me he formado con los estudios de Magisterio & 8 & 17 \\
Me he formado adecuadamente con los estudios de Magisterio & 6 & 13 \\
Me he formado en la práctica, por experiencia & 4 & 8 \\
Formación inicial y formación permanente & 1 & 2 \\
Formación inicial y práctica & 1 & 2 \\
NC & 28 & 58 \\
\hline
\end{tabular}

Tabla 2. Importancia, utilización y formación que declaran los maestros en activo en la entrevista sobre las ideas de los alumnos.

\section{Conclusiones}

Para los maestros encuestados la utilización de las ideas de los alumnos no están entre los indicadores de competencias ni más importantes ni más utilizados, y tampoco consideran que hayan sido especialmente bien formados, y con diferencias significativas con los futuros maestros.

Por otro lado, la mayoría de los maestros entrevistados declaran que es importante utilizar las ideas de los alumnos, tanto por razones de "respeto a las ideas" como de importancia para el aprendizaje, sin olvidar que el 19\% declara que ser competente para utilizar las ideas de los alumnos no es muy relevante para la profesión.

Asimismo, para los maestros entrevistados, las ideas de los alumnos tienen un significado muy diverso: son opiniones, experiencias, errores, conocimientos previos (el nivel de conocimientos que tienen) e incluso una manifestación de la motivación, interés y participación. Ante semejante polisemia, las ideas de los alumnos se tienen en cuenta solo al iniciar el proceso de enseñanza-aprendizaje. En definitiva, los maestros entrevistados se sitúan en niveles de escasa complejidad sobre las ideas de los alumnos, incluso, en niveles por debajo del que denominábamos nivel inicial. En el Gráfico 2 se representan estos niveles, siguiendo las orientaciones de Porlán et al. (2011). 


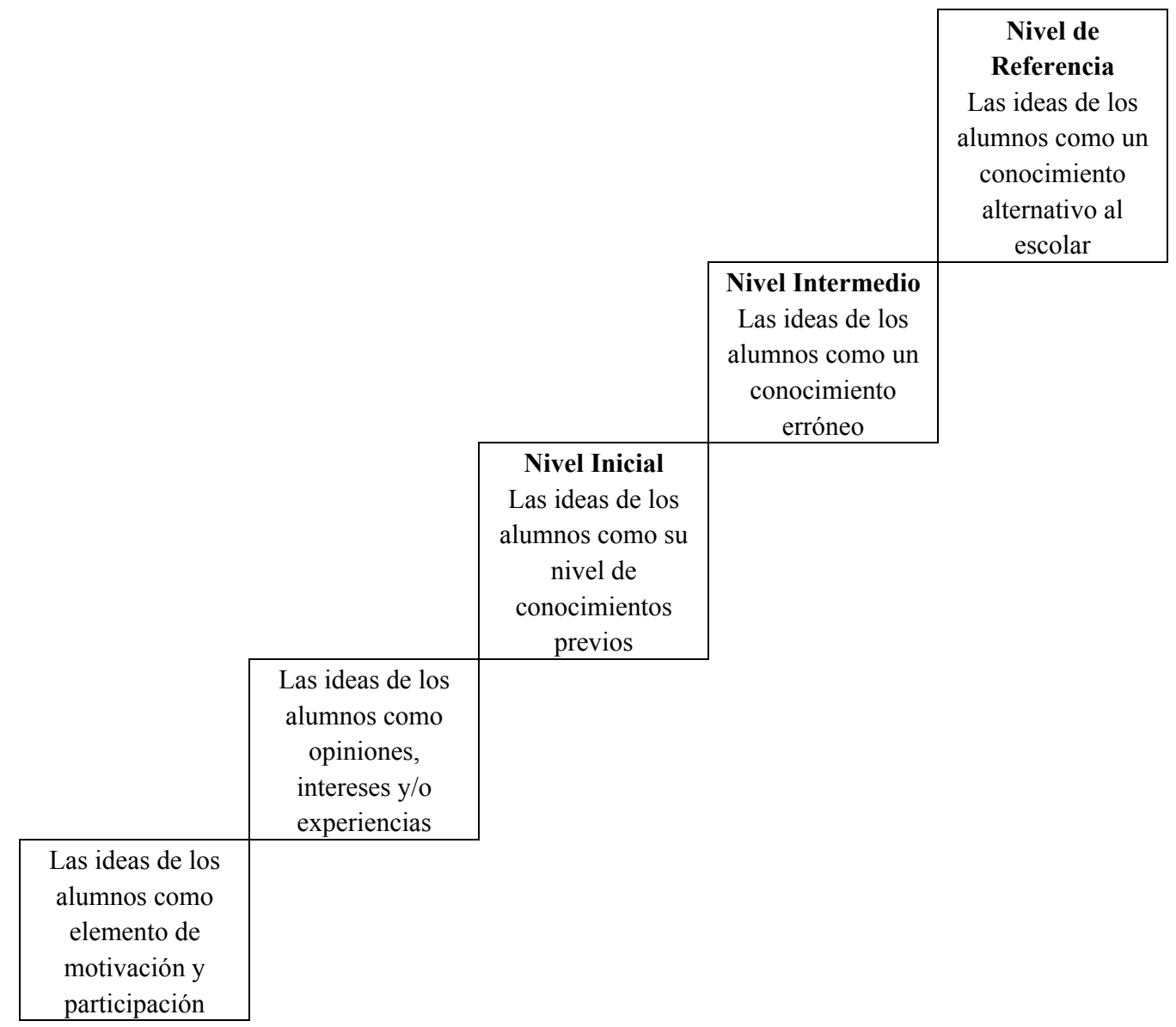

Gráfico 2. Niveles de complejidad sobre las ideas de los alumnos

Estos planteamientos están lejos de una orientación constructivista del proceso de enseñanza-aprendizaje, lejos del modelo que sirve de referencia en la formación inicial de maestros (Duit y Treagust, 2003), y que otorga valor epistemológico a las ideas de los alumnos como un conocimiento alternativo al conocimiento escolar.

Como formadores de profesores, debemos entonces procurar que los futuros maestros sean competentes para detectar las ideas de los alumnos sobre los contenidos escolares, para analizar la gran diversidad de ideas que presentan los alumnos y para poder establecer grupos de ideas comunes, o si se prefiere niveles de competencia, con los que trabajar en el aula, dentro de un modelo de investigación escolar " (Informe Rocard, 2007) en el que se trabaja con las ideas de los alumnos durante todo el proceso de enseñanza-aprendizaje y no solo al inicio para "saber su nivel o sus errores". 
Pero este tipo de formación no es algo aislado, al margen de lo que se denomina aprender a enseñar contenidos (Martín del Pozo, 2007). Debe hacerse integrada en la formación en los contenidos que habrán de enseñar. Como ya indicábamos en un estudio sobre el dominio de los contenidos como competencia profesional (Martín del Pozo et al., 2010), no puede hacerse al margen del análisis y la reflexión sobre asuntos tales como: ¿qué contenidos se prescriben en el currículo de Primaria? ¿qué contenidos incluyen los libros de texto? ¿qué sabemos por nuestra formación escolar? ¿cómo ha evolucionado el conocimiento de un contenido a lo largo de la historia? ¿qué ideas tienen los alumnos de Primaria sobre dicho contenido? ¿cómo evolucionan? ¿qué actividades son más potentes para mejorar las ideas de los alumnos? ¿qué contenidos seleccionar? ¿cómo pueden tener sentido para los alumnos?

Y ello solo es posible dentro de un enfoque integrado de las competencias profesionales (Navío, 2005) y de un modelo simultáneo de formación (Esteve, 2004) en el que los contenidos, su enseñanza y aprendizaje se trabajan como un todo y al mismo tiempo. Como señala Zabalza $(2004$, p.56) el reto es pasar "de la formación dirigida a la asimilación o acumulación de conocimientos a la formación dirigida a la acomodación y construcción de saberes prácticos.”

\section{Referencias bibliográficas}

ANECA (2004). Libro Blanco. Título de Grado en Magisterio. Recuperado de http://www.aneca.es/media/150404/libroblanco_jun05_magisterio1.pdf, 7 de febrero de 2011.

BARDIN, L. (1977). Analyse de contenu. Paris: Presses Universitaires de France. (Trad. cast. Análisis de contenido. Madrid: Akal, 1986).

CUBERO, R. (2005). Perspectivas constructivistas. La intersección entre el significado, la interacción y el discurso. Barcelona: Graó.

DE-JUANAS, A., FERNÁNDEZ, P., MARTÍN DEL POZO, R., GONZÁlEZ, M., PESQUERO, E. y SÁNCHEZ, E. (2009). Comparative study of the evaluation of professional competencies by experienced and trainee Spanish primary teachers. European Journal of Teacher Education, 32(4), 437-454.

DRIVER, R., GUESNE, E. y TIBERGHIEN, A. (1989). Ideas científicas en la infancia y la adolescencia. Madrid: Morata-MEC.

DRIVER, R., SQUIRES, A., RUSHWORTH, P. y WOOD-ROBINSON, V. (1999). Dando sentido a la ciencia en Secundaria. Investigaciones sobre las ideas de los niños. Madrid: Visor-Aprendizaje.

DUIT, R. y TREAGUST, D. (2003). Conceptual change: a powerful framework for improving science teaching and learning. International Journal of Science Education, 25(6), 671-688. 
ESTEVE, J.M. (2004). La profesión docente en Europa: perfil, tendencias y problemática. La formación inicial. Revista de Educación, 340, 19-40.

FURIÓ, C., SOLBES, J. y CARARSCOSA, J. (2006). Las ideas alternativas sobre conceptos científicos: tres décadas de investigación. Alambique, 48, 64-77.

GARCÍA, M.B., MATEOS, M. y VILANOVA, S.L. (2011). Contenido y naturaleza de las concepciones de profesores universitarios de biología sobre el conocimiento científico. Revista Electrónica de Enseñanza de las Ciencias, 10 (1), 23-39.

GIORDAN, A. (1989). Representaciones sobre la utilización didáctica de las representaciones. Enseñanza de las Ciencias, 7(1), 53-62.

GIORDAN, A. y VECCHI, G. (1995). Los orígenes del saber. Sevilla: Díada.

GONZÁLEZ BALLESTEROS, M., PESQUERO FRANCO, E., SÁNCHEZ MARTÍN, E., DE-JUANAS, A., FERNÁNDEZ LOZANO, P. y MARTÍN DEL POZO, R. (2010). El punto de vista de los estudiantes en prácticas sobre las competencias profesionales de los maestros de primaria. Investigación en la Escuela, 72, 71-83.

HAEFNER, L.A. y ZEMBAL-SAUL, C. (2004). Learning by doing? Prospective elementary teachers' developing understandings of scientific inquiry and science teaching and learning. International Journal of Science Education, 26(13), 16531674.

JONES, M., CARTER, G. y RUA, M. (1999). Children's concepts: tools for transforming science teachers' knowledge. Science Education, 83(5), 545-557.

JORAM, E. y GABRIELE, A.J. (1998). Preservice teacher's prior beliefs: transforming obstacles into opportunities. Teaching and Teacher Education, 14(2), 175-191.

LÓPEZ, J.I. (1994). El pensamiento del profesor sobre el conocimiento de los alumnos. Investigación en la Escuela, 22, 59-66.

MARTÍN DEL POZO, R. (2007). Aprender para enseñar ciencias en Primaria. Sevilla: Díada.

MARTÍN DEL POZO, R., FERNÁNDEZ, P., GONZÁLEZ, M. y DE-JUANAS, A. (2010) El dominio de los contenidos básicas: competencia profesional y formación inicial de maestros [doi: 10-4438/1988-592X-RE-2011-360-115], Revista de Educación, 360.

MEYER, H., TABACHNICK, R., HEWSON, P., LEMBERGER, J. \& PARK, H. (1999). Relationships between prospective elementary teachers' classroom practice and their conceptions of Biology and of Teaching Science. Science Education, 83, 323-346.

MORRISON, J.A. \& LEDERMAN, N.G. (2003). Science teachers' diagnosis and understanding of students' preconceptions. Science Education, 87(6), 849-867.

NAVÍO, A. (2005). Propuestas conceptuales en torno a la competencia profesional. Revista de Educación, 337, 213-234. 
OLIVA, J.M. (1999a). Algunas reflexiones sobre las concepciones alternativas y el cambio conceptual. Enseñanza de las Ciencias, 17 (1), 93-177.

OLIVA, J.M. (1999b). Ideas para la discusión sobre el cambio conceptual. Enseñanza de las Ciencias, 17(1), 115-117.

ORDEN ECI/3857/2007, de 27 de diciembre, por la que se establecen los requisitos para la verificación de los titulos universitarios oficiales que habiliten para el ejercicio de la profesión de Maestro en Educación Primaria. Recuperado de http://www.boe.es/boe/dias/2007/12/29/pdfs/A53747-53750.pdf, 16 de marzo de 2012.

ORDEN ECI/2211/2007, de 12 de julio, por la que se establece el curriculo y se regula la ordenación de la Educación Primaria. Recuperado de http://www.boe.es/boe/dias/2007/07/20/pdfs/A31487-31566.pdf, 24 de abril de 2012.

PARK, H., HEWSON, P. W. \& LEMBERGER, J. (2010). The interactions of conceptions of teaching science and environmental factors to produce praxis in three novice teachers of science. Research in ScienceEducation, 40, 717-741.

PERRENOUD, Ph. (2004). Diez nuevas competencias para enseñar. Barcelona: Graó.

PESQUERO, E., SÁNCHEZ, E., GONZÁlEZ, M., MARTÍN DEL POZO, R., GUARDIA, S., CERVELLÓ, J., FERNÁNDEZ, P., MARTÍNEZ, M. y VARELA, P. (2008). Las competencias profesionales de los Maestros de Primaria. Revista Española de Pedagogía, 241, 447-466.

PORLÁN, R., MARTÍN DEL POZO, R., RIVERO, A., HARRES, J., AZCÁRATE, P. y PIZZATO, M. (2011) El cambio del profesorado de ciencias II: Itinerarios de progresión y obstáculos en estudiantes de Magisterio. Enseñanza de las Ciencias, 29(3), 413-426.

POSADA, J.M. (2000). El estudio didáctico de las ideas previas. En: F.J. PERALES y P. CAÑAL (dir.). Didáctica de las Ciencias Experimentales. Alcoy: Marfil.

POZO, J.I., SANZ, A., GÓMEZ, M.A. y LIMÓN, M. (1991). Las ideas de los alumnos sobre la ciencia: una interpretación desde la Psicología cognitiva. Enseñanza de las Ciencias, 9(1), 83-94.

POZO, J.I. y GÓMEZ CRESPO, M.A. (1998). Aprender y enseñar ciencia. Madrid: Morata.

SHULMAN, L.S. (1987). Knowledge and teaching: Foundations for the new reform. Harvard Educational Review, 57, 1-22.

ROCARD, M. (President). (2007). Science Education NOW: A Reviewed Pedagogy for the Future of Europe. Directorate General for Research Science, Economy and Society. Belgium: European Comissión. 
TREAGUST, D.F. y DUIT, R. (2008). Conceptual change: a discussion of theoretical, methodological and practical challenges for science education. Cultural Studies in Science Education, 3, 297-328.

Von GLASERFELD, E. (1993). Questions and answers about radical constructivism. In: K. TOBIN (Ed.). The practice of constructivism in science education. Washington, DC: American Association for the Advancement of Science.

ZABALZA, M.A. (2004). Buscando una nueva hoja de ruta en la formación del profesorado. Revista de Educación, 340, 51-58.

\section{Correspondencia con los autores:}

Rosa MARTÍN DEL POZO

Facultad de Educación-CFP. Universidad Complutense de Madrid

C/Rector Royo Villanova s/n. 28040 Madrid

e-mail: rmartin@edu.ucm.es

Teléfono: 913946249

Ángel DE-JUANAS OLIVA

Facultad de Educación. Universidad Nacional de Educación a Distancia

C/Juan del Rosal 14. 28040 Madrid

e-mail: adejuanas@edu.uned.es

Teléfono: 913986979 